\title{
Die Bienen des armen Mannes in Antike und Mittelalter
}

\author{
GERNOT KRAPINGER
}

Die pseudoquintilianischen Declamationes maiores bilden ein Corpus aus 19 fingierten Gerichtsreden. Ebenso wie die aus 145 Stücken bestehende Sammlung der Declamationes minores ist es unter dem Namen Quintilian überliefert, beide Corpora werden dem berühmten Redelehrer allerdings einhellig abgesprochen. ${ }^{1}$

Die declamatio XIII, die den Titel „Apes pauperis“ trägt, ${ }^{2}$ ist eine der in der Forschung am fleißigsten bearbeiteten ${ }^{3}$ Reden aus der lange ein philologisches Schattendasein fristenden Sammlung, die wahrscheinlich aus dem Ende des 2. Jh.

1 Eine hervorragende Einführung in das gesamte Textcorpus bietet N. Hömke, Gesetzt den Fall, ein Geist erscheint. Komposition und Motivik der ps-quintilianischen Declamationes maiores X, XIV und XV, Heidelberg 2002, 9-55; eine eingehende Text- und Forschungsgeschichte zu den Maiores hat jüngst A. Stramaglia erstellt: Le Declamationes maiores pseudo-quintilianee: genesi di una raccolta declamatoria e fisionomia della sua trasmissione testuale, in: E. Amato (Hrsg.), Approches de la Troisième Sophistique. Hommages à Jacques Schamp. Collection Latomus, volume 296, Bruxelles 2006, 555-576.

2 Im Folgenden „DM 13“ oder „Apes“.

3 Kommentiert wird die Rede schon von B. Pasqualigo, Le api del povero. Declamazione di M. Fabio Quintiliano, Venezia 1734 (mit italienischer Übersetzung) und L. Patarol, M. Fabii Quintiliani Declamationes, Cum earumdem Analysi, \& Adnotatiunculis Difficiliores, \& conditiores sensus explicantibus. In singulas praeterea declamationes Antilogiae. Auctore Laurentio Patarol, in: Id., Opera Omnia quorum pleraque Nunc primum in lucem prodeunt, II, Venetiis 1743, 93402; in jüngerer Zeit kommentierten die Apes M. Winterbottom, Roman Declamation. Extracts edited with commentary, Bristol 1980, 38-52 (Text) bzw. 92-98 (Kommentar) und G. Krapinger, [Quintilian] Die Bienen des armen Mannes (Größere Deklamationen, 13), Cassino 2005; eine Gesamtausgabe der 19 pseudoquintilianischen Declamationes maiores hat L. Håkanson, Stuttgart 1982 (Teubner) vorgelegt; vom Gesamtcorpus gibt es eine englische (L. Sussman, The Major Declamations Ascribed to Quintilian. A Translation, Frankfurt a. M. et al. 1987) und eine italienische Übersetzung (R. L. Pagliaro, Pseudo-Quintiliano. Declamationes XIX Maiores. Con proposta di traduzione in CD-Rom, Napoli 2004 [CD-Rom, 2. Auflage 2006]; Editionen der Minores haben D. R. Shackleton Bailey (M. Fabii Quintiliani declamationes minores, Stuttgart 1989) und M. Winterbottom (The Minor Declamations Ascribed to Quintilian. Ed. with comm., in: Texte und Kommentare, Berlin/New York 1984) besorgt, Shackleton Bailey hat kurz vor seinem Tod noch eine englische Übersetzung in zwei Bänden vorgelegt (Quintilian. The Lesser Declamations [Loeb Classical Library 500-501], Cambridge, Mass. 2006). 
n. Chr. stammt. ${ }^{4}$ Das argumentum dieser „Prunkrede“ ${ }^{\text {(5 }}$ führt uns in das Thema des fiktiven Rechtsfalles ein:

Damni per iniuriam dati sit actio. Pauper et dives in agro vicini erant iunctis hortulis. Habebat dives in horto flores, pauper apes. Questus est dives flores suos decerpi ab apibus pauperis. Denuntiavit, ut transferret. Illo non transferente flores suos veneno sparsit. Apes pauperis omnes perierunt. Reus est dives damni iniuria dati.

„Es möge die Klage wegen widerrechtlicher Schädigung gewährt werden: Ein Armer und ein Reicher waren, da sie auf dem Lande angrenzende Gärten besaßen, Nachbarn. Der Reiche hatte im Garten Blumen, der Arme Bienen. Der Reiche beklagte sich, dass seine Blumen von den Bienen des Armen abgefressen würden. Er gab die Anweisung, die Bienen an einen anderen Ort zu bringen. Als jener dies nicht tat, besprühte er seine Blumen mit Gift. Die Bienen des Armen gingen allesamt zugrunde. Der Reiche ist der widerrechtlichen Schädigung angeklagt.“

Der antike Prunkredner lässt nun ein buntes Potpourri von literarischen Anspielungen an die augusteische Dichtung, von Topoi der Popularphilosophie und von Juristenparodie folgen, Sentimentales, Mitleid und Empörung ob der Bienentötung, angeheizt noch durch die brutale Vernichtung von blühenden Blumen, wird aufgelockert durch juristisches Geplänkel um Nothilfe und Kausalität, Mord und Giftmischerei, ein rhetorisches Schaustück von über 20 Teubnerseiten im thematischen Spannungsfeld der zeitlos gesellschaftlich virulenten Antinomie zwischen Arm und Reich. Der Arme begehrt die Verurteilung des Reichen wegen Sachbeschädigung. Er weist auf seine Armut, Ohnmacht und Unschuld hin und beklagt, mit den Bienen seine materielle und seelische Lebensgrundlage verloren zu haben (§1). In der narratio ( $\S 2-6)$ beschreibt er sein Landgut und sein Leben darauf, fern vom Getriebe der Welt, und die harten Lebensbedingungen des für den beschwerlichen Ackerbau zu alten armen Mannes. Die Freude an den Bienen linderte sein hartes Los. Durch Landflucht und durch die Ausweitung des Großgrundbesitzes ist der Arme zum einzigen Nachbarn des Reichen geworden, dessen Ländereien sein ihm noch verbliebenes Gärtchen einkreisen. Der Arme missachtet den Befehl des Reichen, seine Bienen von seinem Garten fernzuhalten, weshalb der Reiche Gift auf seine Blumen streut und so die Bienen des Armen qualvoll umbringt. Die argumentatio (§ 7) gliedert sich in die propositio, in der der Arme auf vorsätzliche Schadenszufügung, begangen durch die Tötung der Bienen als

$4 \mathrm{Zu}$ den kontrovers diskutierten Problemen wie Datierung, Verfasserschaft und Einheitlichkeit der DM vgl. A. Stramaglia, [Quintiliano] I gemelli malati: Un caso di vivisezione (Declamazioni maggiori 8), Cassino 1999, 33; C. Schneider, [Quintilien] Le soldat de Marius (Grandes déclamations 3), Cassino 2004, 34-38.

5 Winterbottom, Roman Declamation (s. Anm. 3), 37 nimmt als Verfasser Rhetoriklehrer an, die mit ihren Reden Werbung für ihren Unterricht betreiben wollten. 
seines Eigentums, plädiert, in die partitio, in der der Redner festhält, dass ihm ein Schaden entstanden sei und diesen der Reiche vorsätzlich und ohne Vorliegen eines entschuldigenden Notstands zugefügt habe, und schließlich in die refutatio ( $\S ~ 8-14)$, in der der Arme auf mögliche Einwände des Reichen eingeht: Auf das Argument, an Bienen könne man kein Eigentum begründen, da sie freie, fliegende Tiere seien, über die man keine Gewalt habe, kontert der Redner spitzfindig: Das $\mathrm{zu}$ verlieren, was zu haben ein Gewinn ist, stellt einen Schaden dar. Die Bienen sind in seinem Besitz geboren, er verliert das Eigentum an ihnen auch nicht, wenn sie ausfliegen. Da der Besitz an der Frucht der Bienen, dem Honig, unstreitig ist, sei dies auch der Besitz an den Honigproduzenten selbst. Auf den gedachten Einwand des Reichen, der inkriminierte Sachverhalt habe sich auf seinem Grund und Boden abgespielt, wo ihm die Bienen Schaden zufügten, macht der Arme geltend: Der Ort kann nicht entschuldigen, auch bei Raub und Mord ist der Ort der Begehung unerheblich; bei der großen Ausdehnung seines Grundbesitzes stünde der Reiche sonst außerhalb der Rechtsordnung. Wenn der Reiche durch die Bienen tatsächlich geschädigt worden ist, steht ihm eine Klage offen; Selbsthilfe aber ist unerlaubt. Der Reiche wurde aber nicht geschädigt, denn die Blumen, auf die sich die Bienen setzten, leben nur eine sehr kurze Zeitspanne lang, bevor sie ohnedies zugrunde gehen; außerdem ist das, was sie den Blüten entnehmen, so wenig, dass niemand es merkt. Ferner geben die Bienen den Blumen reichlich Ersatz für das, was sie ihnen entnehmen; die Selbsthilfe des Reichen war exzessiv, noch dazu ist er Nutznießer der Honiggewinnung des Armen, da er davon jährlich einen Anteil erhält. Auf das letzte denkbare Entschuldigungsargument des Reichen, die Bienen hätten sich von selbst auf die mit Gift besprühten Blüten begeben, seien also von selbst in den Tod gegangen, erwidert der Arme, dass es auf die äußeren Umstände nicht ankomme, maßgeblich seien die Schädigungsabsicht des Täters und der Eintritt des Schadenfalles. Die peroratio beschließt die Rede mit einer pathetisierenden Rekapitulation des Geschilderten $(\S 15)$ und einem umfangreichen und Mitleid heischenden Bienenlob ( $\S 16-19)$.

Das „Nachleben“ der Apes war geringer als das einiger anderer Reden des umfangreichen Corpus der kaiserzeitlichen Rhetorikschule. ${ }^{6}$ Lediglich Hierony-

6 Vgl. etwa zur Declamatio maior I (Paries palmatus) G. Krapinger, Vives' Antwort auf Ps.Quintilians „Paries palmatus“: Die Deklamation „Pro Noverca“, Text, Übersetzung und Erläuterungen, in: B.-J. und J.-P. Schröder (Hrsg.), Studium declamatorium. Untersuchungen zu Schulübungen und Prunkreden von der Antike bis zur Neuzeit (Joachim Dingel zum 65. Geburtstag), München/Leipzig 2003, 289-333, zur Declamatio maior III (Miles Marianus) C. Schneider, Littérature et propagande dans le recueil des Grandes déclamations pseudo-quintiliennes au IVe siècle de notre ère: l'exemple du Miles Marianus (Ps.-Quint., Decl. Mai. III), in: F. E. Consolino (éd.), Letteratura e propaganda nell'Occidente latino da Augusto ai regni romanobarbarici. Atti del convegno internazionale (Arcavacata di Rende, 25-26 maggio 1998), Roma 2000, 45-46 sowie T. Haye, Oratio. Mittelalterliche Redekunst in lateinischer Sprache, Leiden 1999, 
mus zitiert die Sentenz aus $\S 2$, wonach dem Neid kein Opfer entgeht (vgl. Vit. Malch. Mon. 16 [PL 23, Sp. 58b: O nihil tutum apud diabolum! ... Sic quoque latentem me invenit invidia] und Hebr. quaest. in libr. Genes., praef. [CC SL 72, S. 1, 21-22]: Me vero procul ab urbibus, foro, litibus, turbis remotum, sic quoque [ut Quintilianus ait] latentem invenit invidia ${ }^{7}$ ). Dass auch unsere die genannten zwei Elemente auf einzigartige Weise vereinigende Rede im Mittelalter nicht vergessen wurde, ${ }^{8}$ bezeugt das Gedicht Versus de quodam paupere. ${ }^{9}$ A. Beaugendre, der erste Herausgeber, hielt unser Gedicht für ein Werk Hildeberts von Lavardin. ${ }^{10}$ Erst B. Haréau meldete daran Zweifel an: „Ce sont des hexamètres dactyliques, et il y en a beaucoup de semblables dans la même pièce. Eh bien, si la Muse d'Hildebert s'est exercée sur bien des rythmes, elle a, comme il paraît, méprisè celui-là. On sait, en effet, positivement qu'Hildebert a seulement composé, durant tout le cours de sa vie, quatre vers dactyliques, qui sont bien différents de ceux qu'on vient de lire“; $;{ }^{11}$ der Gelehrte tendiert zu dem Zisterzienser Serlo von Wilton (gest. um 1181), ${ }^{12}$ J. Öberg nimmt allerdings das Gedicht nicht in seine Serlo-Ausgabe auf, sondern schreibt es eher Bernardus Silvestris zu. ${ }^{13}$ Wir halten uns an die Ausgabe von J. J. Bourassé (PL 171, Sp. 1400b-1402b).

191-212; zur Declamatio maior VIII (Gemini languentes) J. Werner, Beiträge zur Kunde der lateinischen Literatur des Mittelalters aus Handschriften gesammelt. Zweite durch einen Anhang vermehrte Ausgabe, Aarau 1905, 55-58, R. R. Edwards, Poetic Invention and the Medieval causae, in: Mediaeval Studies 55 (1993) 196-210; zur Declamatio maior IX (Gladiator) vgl. B. Smalley, English Friars and Antiquity in the Early Fourteenth Century, Oxford 1960, 180f. (zur Gladiator-Rezeption Robert Holcots); zur Declamatio maior X (Mathematicus) Edwards, 183196; C. Ratkowitsch, Astrologie und Selbstmord im Mathematicus. Zu einem Gedicht aus dem Umkreis des Bernardus Silvestris, in: WSt 112 (1999) 175-229.

7 S. jetzt C. Schneider, Lactance, Jérôme et les recueils de déclamations pseudo-quintiliens, in: Autour de Lactance. Hommages à P. Monat, Besançon 2003, 43-44.

8 Zur Verbreitung der pseudoquintilianischen Declamationes vgl. J. E. Bliese, Medieval Rhetoric: Its Study and Practice in Northern Europe from 1050 to 1250, Ph. D. dissertation Univ. Kansas 1969, 52-66; H. Buttenwieser, The Distribution of the Manuscripts of the Latin Classical Authors in the Middle Ages, unpublished Ph. D. dissertation, Univ. of Chicago, 1930, 139.

9 Zur immensen Bedeutung der Deklamatorik für die mittelalterliche Poesie vgl. R. R. Edwards, Poetic Invention and the Medieval causae, in: Mediaeval Studies 55 (1993) 183-217.

10 A. Beaugendre, Venerabilis Hildeberti primo Cenomanensis episcopi, deinde Turonensis archiepiscopi opera tam edita quam inedita, Paris 1708, 1327-29.

11 B. Haréau, Les mélanges poétiques d'Hildebert de Lavardin, Paris 1882, 38.

12 B. Haréau, Notices et extraits de quelques manuscrits latins de la Bibliothèque Nationale 31 , $1884,126$.

13 Serlon de Wilton. Poèmes latins, Stockholm 1965, 19 A. 33: „On penserait à Bernard Silvestre plutôt qu'à Hildebert“, - wir überlassen weitere Versuche einer Klärung den berufeneren Mediävisten; zur Verfasserfrage und zum Überlieferungsbestand vgl. G. Gröber, Übersicht über die Literatur von der Mitte des VI. Jahrhunderts bis zur Mitte des XIV. Jahrhunderts, Strassburg 1902, 391 (= Nachdruck München 1963); A. G. Rigg, A History of Anglo-Latin Literature 1066-1422, Cambridge 1992, 70-72; M. Manitius, Geschichte der lateinischen Literatur des Mittelalters, III (Vom Ausbruch des Kirchenstreites bis zum Ende des 12. Jh.), München 1931, 907. 
Ein Armer beklagt beredt und wortspielreich sein bedrückendes Schicksal, da ein reicher Nachbar ihm Kühe und Schafe genommen und die Bienen vergiftet hat. Ausgehend vom Text wollen wir die intertextuelle Verwertung der Apes pauperis durch unseren mittelalterlichen Verseschmied untersuchen.

\section{Text}

Vestra peritia dum regit omnia, sidera tangit ${ }^{14}$ Dum domat effeta, mitigat aspera, fortia frangit. Non odio, non fit pretio, non fit prece cetus; Justitiae rigor et fidei vigor omnibus aequus, Non pretium tulit auxilium qui dona dedere; Nec nocuit qui nihil potuit, qui donat habere. Hinc fuit unica spes mea civica iura ${ }^{15}$ subire,

$8 \quad$ Et quasi brutum iurgia tutum fecit inire.

9 Sit docilis mihi, sit facilis mens ${ }^{16}$ vestra, precamur,

10 Forsitan, his patet, et tamen hic latet unde queramur

11 Noxia pestis, littera testis, dives avarus.

12 Dives avarus quam sit amarus, quam malus hostis

13 Sentio plus nimio, sed vos tamen hic bene nostis,

14 Corpore maximus, ille pigerrimus, ille Pilatus

15 Ille deorum spector, eorum templa paratus,

16 Ignibus urere, cunctaque spargere membra ${ }^{17}$ senatus.

17 Exitio nostri nimio vestro quoque natus,

18 Me male percutit, et satis intulit aspera damna

19 Aspera tristitia, quae sibi vilia, sed mihi magna;

20 Namque boves paucas et oves spe lactis alebam;

21 Hinc ego victum vix et amictum vestis habebam:

22 Flebat euntes et redeuntes invidus illas

23 Pergere laetas, atque repletas lacte mamillas

24 Quando videbat, deficiebat mente, quod ille

25 Inde careret, cum vel haberet millia mille.

14 Vgl. Ov. epist. 2, 2, 9f. (non ego concepi, si Pelion Ossa tulisset, / clara mea tangi sidera posse manu); 2, 5, 57 (huic cum tu placeas et vertice sidera tangas) et pass.

15 Hor. epist. 1, 3, 23f. (seu linguam causis acuis seu civica iura / respondere paras seu condis amabile carmen).

16 Vgl. Ov. ars 1, 358 (quo facilis dominae sit et apta capi).

17 Ov. Ibis v. 435 (spargantur membra per agros); Sen. Phoen. 448 (haec membra passim spargite ac divellite), Lucan. 5, 684 (invitis spargenda dabas tua membra procellis). 
26 Has sibi vendi poscit, habendi ductus amore.

27 Non volo vendere vi, prece, munere, sive timore:

28 Ergo superbia divitis impia mota furore

29 Dicere turpia coepit, et omnia plena pudore;

30 Tunc mihi verbera, tunc gregis impia damna minatus,

31 Clausit $^{18}$ euntibus et redeuntibus arte meatus;

32 Scilicet atria qua pecori via sola patebat,

33 Iam gregibus dominoque cibus sic deficiebat.

$34 \quad$ Quid facerem? Quid me sinerem pecudesque perire?

35 Grandis inedia divitis atria cogit adire.

$36 \quad$ Nam scio vendere quam male perdere tutius esse.

37 Tunc facio mihi quod ratio probat esse necesse.

$38 \quad$ Vendo quidem sibi, quandoquidem prohibet retinere

39 Mereor apes quibus ille dapes nequeat prohibere.

$40 \quad$ Has quoque dum videt invidus invidet, utque necari

$41 \quad$ Possit in ortis pocula mortis ${ }^{19}$ sparsit amara.

$42 \quad$ Mane sequenti sole recenti ${ }^{20}$ fracta labore

$43 \quad$ Atria cerea linquit apes mea mellis amore.

44 Rex ut eat dignum, properat prior ad loca nota;

$45 \quad$ Inde duces, heu! non reduces, nunc curia tota.

46 Ut fuerant solitae redeunt non absque canore,

$47 \quad$ Utque solo resident, solo moriuntur odore.

$48 \quad$ Qualia corpora, qualia pectora tunc abiere!

49 Agmina fortia, proh dolor ${ }^{21}$ ! omnia procubuere;

$50 \quad$ Pluria corpore, maxima pectore, terrica moles.

51 Quid tibi parvula fecit apecula coelica proles?

52 Sic bove, sic ape, sic et dape me spoliavit;

53 Vos quoque laedere, iuraque spernere non dubitavit.

$54 \quad$ Quid faciam miser? An fugiam? Si fugero, victum

$55 \quad$ Non habeo, neque si maneo, panem vel amictum.

$56 \quad$ Ha! miseri patris pueri, vos quid facietis?

57 Saepius interius mihi corda dolore movetis.

18 Serv. in Verg. Aen. 6, 287 (Hercules ... aquae clausit meatus); Macrob. sat. 7, 11, 9 (musculi quibus claudebantur retrimentorum meatus), Hildeg. Bing. causae et curae 2 (162, $3 \mathrm{ff}$.: tempestates et procellae malorum humorum ... meatum sanginis claudunt).

19 Cic. Cluent. 31 (exhausto illo poculo mortis).

20 Pers. sat. 5, 54 (sub sole recenti).

21 Die Apostrophe an dolor verzeichnen wir schon bei Cic. Tusc. 2, 61; vgl. noch Stat. Theb. 1, 77, Auson. Epitaph. (carm. 15, 4), Serv. Comm. in artem Donati 420, 19, Claud. in Ruf. 5, 55. 
$58 \quad$ Non teneris etiam laceris dat frustula panis

59 Mater anus, vacuaque manus, stupet alius [f. alvus] inanis.

60 Sed ego miser haec retego, nil ille movetur.

61 Plus doleo, plus damna fero, plus laetus habetur.

62 Vos igitur quid nunc agitur ne spernite, quaeso,

63 Indicioque pio misero succurrite laeso.

64 Namque superbia divitis impia ni teneatur,

65 Dicere turpia, vel magis impia non vereatur.

66 Non noceat mihi quod doleat me pauper amari:

67 Imo fleat quod non videat se hac parte beari.

68 Non pretio vos allicio, sed amore paravi,

69 Obsequioque pio retinendos ${ }^{22}$ esse putavi:

$70 \quad$ Nam scitis quoniam meritis retinentur amici

71 Hique iuvandi sunt et amandi non inimici.

72 Exterius velut interius fit lumine caecus:

73 Sic inimicis sicut amicis qui manet aequus

74 Paupere fortior atque potentior ipse quidem sum,

75 Non ego noxius, aut magis impius inde tamen sum.

76 Imposuit mihi quod voluit per me periisse;

77 Dixit apes, armenta, dapes, ait eripuisse:

78 Eripuissem si voluissem, sed nec ademi;

79 Aere dato, pactoque rato mihi vendidit, emi.

$80 \quad$ Iure meo nihil possideo, nolite tacere.

81 Aes habuit, res empta fuit, quis debet habere

82 Haec modo gratia quod pecori via nostra patebat,

83 Et quod in atria plura turpia grex faciebat.

84 Praetereo quod flore meo mel apes faciebat,

85 Intima lilia, pabula mitia ferre solebat,

86 Postea cerea, donaque mellea conficiebat.

87 Non vitem, non humum ditem piger ille colebat;

88 Non ager hordea, nec sibi vinea vina ferebat.

89 Hinc merito, non enim dubito, miser esuriebat,

90 Ac reproborum more proborum lucra dolebat.

91 Praecipue mea triste ferens ea quando videbat.

92 Cur habui pecudes docui quod apes periere.

93 Denique mortis si quis in hortis semina sparsit,

$94 \quad$ Cur mihi verbum pauper acerbum fatus inarsit?

22 Zum Sprachgebrauch vgl. dist. Catonis 1, dist. 34 (221: vincere cum possis, interdum cede sodali, / Obsequio quoniam dulces retinentur amici). 
95 Sunt mihi lilia, prata, rosaria, fructus et arbor

96 Omnia vendere, singula perdere nonne vetabor?

97 Sunt mihi gramina, nescio nomina, res mihi mille,

98 Quae super omnia spargere noxia curve vetet ille?

99 In mea pascua venit apes sua, quam prohibere

100 Si potuit, cur non voluit sua damna cavere?

101 Sed video quod vos adeo lacrymosus adorat,

102 Damna meo quocunque modo reparare laborat,

103 Dum loquitur, dum conqueritur, dum verba colorat ${ }^{23}$

104 Ex odio nostro nimio facundus adorat.

105 Ergo licentia pauperis impia ni teneatur,

106 Turpia dicere, falsaque fingere non vereatur.

Formal belässt der mittelalterliche Versifikator wenig vom antiken Vorbild: Der Umfang ist massiv reduziert, die Sprache gebunden, er ändert wesentliche Grundvoraussetzungen der antiken declamatio. Geht diese von einer nach einem gängigen Muster aufgesetzte lex (damni per iniuriam dati sit actio) aus, auf der eine Gerichtsrede nach allen Regeln der in der Rhetorenschule vermittelten Kunst basiert, so stellen die leoninischen Verse mit Binnen- und Endreim ${ }^{24}$ sich als Streitgedicht eines von einem Reichen in Bedrängnis gebrachten Armen dar, dessen Lamento von rechtfertigenden, oft bis zur Lächerlichkeit überzogenen Einwürfen des reichen Schädigers (vv. 66-79; 81-106) unterbrochen wird. ${ }^{25}$ Auch dies ist den antiken declamationes fremd, in denen der Redner vor Gericht immer der alleinige Sprecher bleibt, lediglich als rhetorische Finesse die gedachten Einwürfe der Gegenseite formuliert und dann bekämpft. Eine forensische Einbettung,

$23 \mathrm{Zu}$ colorare („Farbe geben“, „,mit Tünche überziehen“, „einen Anstrich geben“) als rhetorischem Fachterminus vgl. Sen. contr. 2, 6, 7; 7, 7, 1; Ps.-Quint. decl. min. 285, 1; Winterbottom, Minor Declamations (s. Anm. 3), 400; nebst ThLL III, 2, Sp. 1724, 21-56.

24 S. dazu W. Grimm, Zur Geschichte des Reims, in: Kleinere Schriften von Wilhelm Grimm. Herausgegeben von G. Hinrichs, Gütersloh 1887, IV 244-296; W. Meyer, Gesammelte Abhandlungen zur mittellateinischen Rythmik, Berlin 1905, I 196; P. Klopsch, Einführung in die mittellateinische Verslehre, Darmstadt 1972, 78f.

$25 \mathrm{Zu}$ den verschiedenen Ausprägungen des Sprecherwechsels im Streitgedicht sowie zur mitunter fehlenden Instanz eines Schiedsrichters s. P. Stotz, Conflictus. Il contrasto poetico nella letteratura latina medievale, in: M. Pedroni/A. Stäuble (Hrsgg.), Il genere ,tenzone' nelle letterature romanze delle origini, in: Atti del Convegno internazionale Losanna 13-15 novembre 1997, Sezione d'italiano dell'Università di Losanna, Memoria del tempo, Collana di studi e testi medievali e rinascimentali 15, Ravenna 1999, 165-187, ich zitiere die deutschsprachige Originalfassung hiervon (Beobachtungen zu lateinischen Streitgedichten des Mittelalters: Themen Strukturen - Funktionen), die unter http://www.unizh.ch/mls/onlinepub/stotz-Streitgedichte.htm im Internet zu lesen ist, vgl. bes. $4 \mathrm{ff}$; $7 \mathrm{ff}$. 
wie sie in der lateinischen Deklamatorik gang und gäbe ist, ist dem mittelalterlichen Gerichtsagon ohnedies unbekannt.

Ungeachtet der Verschiedenheit der Anlage und des Aufbaus der zwei Textsorten ${ }^{26}$ erinnert das Gedicht an vielen Stellen nahezu wörtlich an die antike Prunkrede:

Schon v. 1 schlägt mit sidera tangit ein antikes Motiv der Seefahrt an; wir finden hier wohl eine Variante des Motivs für die menschliche Vermessenheit, Elemente miteinander zu verbinden, die die Natur getrennt hat, ${ }^{27}$ ein Gedanke, den DM 13, 16 mit Etiam ratio nostra, quae sub terris lucrum invenit, quae maria inquisitione sua sideribus inmiscuit näher ausführt.

Die Bienen sind Opfer einer noxia pestis (v. 11, vgl. DM 13, 7: inevitabilem pestem) geworden, wodurch der Arme seiner Existenzgrundlage beraubt ist (v. 7: unica spes mea, vgl. DM 13, 1: ab omni spe tuendae paupertatis exclusus). Doch der Reiche hat sich an keinem gewöhnlichen Tier vergriffen! Der Gedanke der Gottähnlichkeit bzw. Gottesnähe der Bienen ist alt. ${ }^{28}$ Einen letzten Rest des bei DM 13, 16 nach dem Vorbild Vergils ${ }^{29}$ ausgestalteten Motivs finden wir in v. 51 mit dem Epitheton coelicola ${ }^{30}$ wieder (Quid tibi parvula fecit apecula coelicula proles?).

Selbst wo der mittelalterliche Dichter den Armen Kühe und Schafe preisen lässt, weckt er Assoziationen mit der liebevollen Schilderung des Bienenlebens in den Apes: Die Beschreibung des in Frohsinn erlebten (v. 23: laetas) und in gleichmäßiger Wiederkehr verlaufenden (v. 22: euntes et redeuntes) Daseins seines Viehs, das einzig der Ernährung des armen Herrn (v. 23: repletas lacte mamillas) geweiht ist, nimmt kräftig Anleihen an der Schilderung des Tagwerks der

$26 \mathrm{Zu}$ den mittelalterlichen Disputen zwischen Arm und Reich und juristischen Scheindisputationen s. H. Walther, Das Streitgedicht in der lateinischen Literatur des Mittelalters, in: Quellen und Untersuchungen zur lateinischen Philologie des Mittelalters, Bd. 5, Heft 5, München 1920, 124-135 (Nachdruck mit einem Vorwort, Nachträgen und Registern von P. G. Schmidt, Hildesheim, 1984); einen hervorragenden Überblick über die Variablen und Konstanten der weitläufigen und vielgestaltigen Gattung des Streitgedichts verschafft Stotz, Conflictus (s. Anm. 25).

27 Vgl. Hor. carm. 1, 3, 21-26 (Nequiquam deus abscidit / prudens Oceano dissociabili / terras, si tamen impiae / non tangenda rates transiliunt vada. / Audax omnia perpeti / gens humana ruit per vetitum nefas).

28 Hymn. Hom. 4, 550-565; Pind. Pyth. I4, 60-61; Xen. Oec. 7, 32; Ios. Fl. ant. Iud. 5, 200; Plut. Pyth. or. 17, 402d; Paus. 9, 40, 1; Ael. nat. an. 1, 11; 5, 13; Philostr. vit. Apoll. 6, 10 (I, S. 214, 31 Kayser).

29 Vgl. Verg. Georg. 4, 220-222 (esse apibus partem divinae mentis et haustus / aetherios dixere; deum namque ire per omnis / terrasque tractusque maris caelumque profundum).

30 Ein Ausdruck, den die Antike bei aller sonstigen Wertschätzung den Bienen nicht zuschreibt, vgl. ThLL III, 73, 44-74, 59. 
Bienen in DM 13, 4: ad cotidiana opera laetius solito agmen effuderat bzw. § 17: duraturus hiemi reponitur victus, et repletis vere cellis tutus annus est.

Die Sonne bestimmt den Tagesablauf der Bienenwelt: Mane sequenti sole recenti (v. 42) reimt unser Dichter, Pseudoquintilian hält sich in DM 13, 9 (At non ipsae domum sua sponte revolant finemque laboris sui sole metiuntur) an Vergil (Georg. IV, 27-28: alas pandere ad aestivum solem); der Arbeitsalltag, überhaupt das ganze Sozialleben gestaltet sich in beiden Fällen in militärischen Formationen, vgl. v. 44: rex, v. 45: duces, v. 49: agmina fortia, stärker akzentuiert diesen Aspekt noch DM 13, (vgl. § 4: ad cotidiana opera laetius solito agmen effuderat; $\S$ 9: apes ... in opus exire ad cotidianum censum laboris non detractare militiam und endlich $\S 17$ : motis pro rege castris procurrere et inire bella mortemque honestam pro duce oppetere [sc. apes]).

Der Erwerb der Bienen ist in beiden Ausbeutungsszenarien der Endpunkt des vom Reichen verursachten wirtschaftlichen Ruins des Armen, die Verse (39ff.) Mereor apes quibus ille dapes nequeat prohibere. / Has quoque dum videt invidus invidet, utque necari / Possit in ortis pocula mortis sparsit amara ensprechen in etwa der Schilderung des letzten Ausweges im Existenzkampf des Bienenbesitzers in DM 13, 2 (Namque ego, iudices, dum fortius opus permisit aetas, terram manibus subegi, et difficultatem labore perdomui, et invito solo nonnihil tamen fecunditatis expressi) und DM 13, 3 (Quid agimus? inquam, undique vallo divitiarum clusi sumus. Hinc hortuli locupletis, hinc arva, inde vineta, hinc saltus; nullus terra[e] datur exitus. Quaeramus animal quod volet. Nam quid apibus invenit natura praestantius? Parcae, fideles, laboriosae. O animal simile pauperibus). Doch es ist keine Habe so gering, dass der Reiche sie dem Armen nicht neidet (v. 40: Has quoque dum videt invidus invidet, vgl. DM 13, 2: Hoc mihi parvulum terrae et humilis tugurii rusticum culmen aequitas animi regna fecerat, satisque divitiarum erat nihil amplius velle. Quid prodest? Sic quoque me latentem invenit invidia).

In beiden Texten beklagt sich der Redner darüber, dass der Reiche ihn durch die rücksichtslose und brutale Expansion seiner Bodenerwerbungen einkreist, sodass er kaum mehr einen Fuß vor den anderen setzen kann, ohne das nachbarliche Grundstück zu betreten: v. 31 (Clausit euntibus et redeuntibus arte meatus) findet in DM 13, 3 (ex omni parte circumiectus divitis ager vix tenuem ad gressus meos semitam dabat ... clusi sumus) eine deutliche Paralelle.

Der Reiche schreitet in provokanter gespielter Skrupulosität nicht ohne Vorwarnung zur endgültigen Vernichtung der wirtschaftlichen Existenz des Armen, v. 30 (Tunc mihi verbera, tunc gregis impia damna minatus) korrespondiert mit DM 13, 13 ('Admonui,' inquit 'et, ut transferres, denuntiavi'). Das Vergiften der Blumen schließlich, das zum Tod der sich am Nektar labenden Bienen führt (v. 41: pocula mortis sparsit amara), verknüpft die antike Formulierung des Besprü- 
hens der Blumen mit Gift durch den reichen Nachbarn (DM 13, 5: sparsit omnibus floribus mortem [sc. dives]) mit dem Passus der Apes, in denen der Redner eine in der Rechtsliteratur bekannte Stelle über die Frage der mittelbaren Verursachung beim Vergiftungstod ${ }^{31}$ diskutiert: Die Bienen hätten sich ja von selbst und ohne Zwang auf seine kontaminierten Blumen gesetzt, so wie jemand, dessen Trank vergiftet worden ist, dadurch dass er sich den Becher selbst zuführt, selbst den finalen Akt der Vergiftungshandlung setzt (DM 13, 14: labiis admovisse pocula). Dieses Argument einer reductio ad absurdum wird vorbereitet durch das abstruse Verteidigungsargument, der Reiche hätte in legaler Selbsthilfe gegen die Blumen- oder Nektarräuber sich nur zur Wehr gesetzt: § 10: 'Ut damnum sit,' inquit (sc. dives) 'iure tamen feci in privato meo', § 12: 'Decerpebant' inquit (sc. dives) 'flores meos', ein Gedanke, den der mittelalterliche Verseschmied in eine Praeteritio gegossen hat: v. 84ff.: Praetereo quod flore meo mel apes faciebat, I Intima lilia, pabula mitia ferre solebat, / Postea cerea, donaque mellea conficiebat.

$\mathrm{Zu}$ den argumentatorischen und motivischen Anklängen an die Apes sind besonders die Verse 64f. Namque superbia divitis impia ni teneatur, / Dicere turpia, vel magis impia non vereatur zu zählen, sie deuten an, was die antike Passage in DM 13, 10 deutlicher ausspricht, wo der Redner die Ohnmacht seiner Armut vor der Arroganz des reichen Nachbarn beklagt und die Richter zu einem Präjudiz gegen Machtmissbrauch auffordert (non sufficit his partibus unus rusticus pauper, obviam publice eundum est et obiciendae adversus nascentem licentiam consensu manus. Credite mihi, maior lite quaestio est. hoc vobis hodie iudicandum est, ubi scelus facere non liceat).

In DM 13, 7 wird die Vertilgung der Bienen damit gerechtfertigt, dass sie auf eigenem Boden geschehen sei, noch dazu an Tieren, die ihrer speziellen Natur nach niemandem gehören (Negat [sc. dives] esse damnum, quod animal liberum et volucre et vagum et extra imperia positum perdiderim. Negat iniuria datum, quod in privato suo, quod eas, quae sibi nocerent, extinxerit); - zumindest das Argument, dass die Bienen an ihrem Tod selbst schuld seien, da sie ja sich selbst auf das Gift gesetzt hätten, macht der Reiche in v. 99f. (In mea pascua venit apes sua, quam prohibere / Si potuit, cur non voluit sua damna cavere?) geltend.

Neben den Parallelen an winkeladvokatorischen Kniffen beider Elaborate decken sich auch die rechtspolitischen Erwägungen, mit denen der Arme jeweils seinem Vorbringen Gewicht verleiht: v. 4 appelliert an die Gleichheit vor dem Gesetz (Justitiae rigor et fidei vigor omnibus aequus) ebenso wie DM 13, 14

31 S. D. Nörr, Causa mortis. Auf den Spuren einer Redewendung, München 1986, 49f.; 192-201, ferner Krapinger, Bienen (s. Anm. 3), 136f. mit Belegen. 
(Tam honestis in hac, ut putamus, aequissima libertate legibus vivimus, ut nobis habere medellam non liceat, vobis habere liceat venena?).

Der Redner der Apes erinnert in $\S 7$ (non de praeterito tantum litigamus; hoc agitur, ut, etiam si quid forte reparavero, iterum diviti liceat occidere) und $\S 10$ (Per fidem vestram, iudices, succurrite exemplo; non sufficit his partibus unus rusticus pauper, obviam publice eundum est et obiciendae adversus nascentem licentiam consensu manus) die Richter daran, dass die Ahndung des von ihm beklagten verbrecherischen Bienenmordes aus einem über den konkreten Einzelfall weit hinausgehenden Interesse geboten ist und berührt damit antike Gedanken über Sinn und Zweck des Strafens; ${ }^{32}$ unser Gedicht spricht mit v. 53 (Vos quoque laedere, iuraque spernere non dubitavit) und v. 64f. (Namque superbia divitis impia ni teneatur, / Dicere turpia, vel magis impia non vereatur) die spezialpräventive Wirkung von Sanktionen an.

Der juristische Aspekt dieser Rede ist nicht der attraktivste an den Apes: Einen beträchtlichen Teil der Rede nimmt das ekphrasisartig eingebaute Bienenlob ein, das sein assoziatives Spiel mit der lateinischen Bienenliteratur treibt, vor allem mit Varro und dem vierten Buch der Georgica Vergils. Neben der Ausschmückung des Gegensatzes zwischen Arm und Reich mit der Problematik der Grundstückskonzentration in den Händen weniger Reicher sowie popularphilosophischer Klischees vom edlen Landmann und glücklichen Asketen wird auf recht unterhaltsame Weise das Arsenal literarischer Topoi der römischen Dichtung ausgebreitet, so etwa die Stadt-Land-Antithese mit einem locus amoenus der Schilderung der Hütte und des Gärtchens des Armen (man vgl. etwa nur § 2: Est mihi paternus, iudices, agellus, sane angustus et pauper, non vitibus consitus... sowie $\S 3$ : Fusus ex proximo fonte rivus trepidantibus inter radiantes calculos aquis utrimque ripa virente praeterfluit. Satis consiti flores et viridis quamvis paucarum arborum coma nascentibus populis prima sedes, unde ego frequenter consertum novae iuventutis agmen ramo gravescente suscepi). Auch unser Gedichtchen trägt dem „en miniature“ Rechnung, indem es neben allerlei affektischen Stilmitteln und miserationes um das herzzerreißende elendigliche Dahinscheiden der vergifteteten Bienen und das unerträgliche Joch der Armut vor allem

32 Vgl. Cic. Inv. 1, 101: Tertius locus est, per quem quaerimus, quidnam sit eventurum, si idem ceteri faciant; et simul ostendimus, huic si concessum sit, multos aemulos eiusdem audaciae futuros; ex quo, quid mali sit eventurum, demonstrabimus; Tull. 8: ea consuetudo non solum ad res privatorum, sed ad summam rem publicam pertinere; Quint. IV, 1, 7; V, 13, 21; ferner Winterbottom, Minor Declamations (s. Anm. 3), 294 zu Ps.-Quint., Decl. min. 244, 6 mit weiteren Belegen. 
bukolische Einsprengsel ${ }^{33}$ einstreut (v. 20f.: Namque boves paucas et oves spe lactis alebam; / Hinc ego victum vix et amictum vestis habebam; v. 95: Sunt mihi lilia, prata, rosaria, fructus et arbor; v. 97: Sunt mihi gramina, nescio nomina, res mihi mille), wodurch die parodistische Stimmungsebene der Apes beibehalten wird.

33 Dabei sei erwähnt, dass neben juristischen Querelen der „Wechselgesang zweier Hirten als ländlicher Sängerwettstreit“" ein weitere Wurzel des Streitgedichts ausmacht, s. Stotz, Conflictus (s. Anm. 25), $2 \mathrm{f}$. 
